\title{
A Study on Modal Fabric using Formic Acid Treatment for K/S, SEM and Fourier Transform Infrared Spectroscopy
}

\author{
J. JEYAKODI MOSES ${ }^{1}$ and GNANAPRIYA ${ }^{2}$ \\ 'Department of Applied Science, PSG College of Technology, Coimbatore 641004, India. \\ ${ }^{2}$ Research and Development Centre, Bharathiyar University, Coimbatore 641045, India. \\ ${ }^{*}$ Corresponding author E-mail: jj_moses2k2@yahoo.co.in
}

http://dx.doi.org/10.13005/ojc/320235

(Received: February 10, 2016; Accepted: March 12, 2016)

\begin{abstract}
Modal is a second generation regenerated cellulosic fiber obtained from wood pulp, particularly from the beech tree wood. Modal was at first introduced by Lenzing AG Company of Austria. Modal is very soft, shiny in nature and silk feel than mercerized cotton with the ability to absorb up to $50 \%$ more water than cotton. In this study, modal fabrics (woven and knitted) are selected and conventionally pretreated and subjected with $98 \%$ formic acid in different concentrations. The formic acid treated modal fabrics were then undergone for different testings, such as, physical properties, wicking, K/S values, fastness properties, SEM studies and FTIR analysis. These treatments on modal fabrics were correspondingly compared with those of cotton for its effectiveness.
\end{abstract}

Keywords: Modal Fabric, Wicking, K/S, SEM, FTIR

\section{INTRODUCTION}

Modal is a semi-synthetic cellulose fibre made by spinning reconstituted cellulose, in this case from beech trees

Modal is a second generation regenerated cellulosic fiber and a variation of rayon. Lenzing started selling modal fibers in 1964. In 1977, Lenzing started using an environmentally friendly bleaching method for pulp for their cellulosic fibers. Modal is amazingly comfortable to wear. Measurements of the softness factor show that modal is twice as soft as cotton. The finer the fibre, the finer the textile becomes. The beechwood fibre gives modal a fineness comparable to natural silk and makes modal so silky soft on the skin ${ }^{1,2}$. Modal is about $50 \%$ more hygroscopic, or water-absorbent, per unit volume than cotton. It's designed to dye just like cotton and is color-fast when washed in warm water. Even after repeated washing, modal remains absorbent, soft and supple. Modal fibers have found a wide variety of uses in clothing, outerwear and household furnishings. The colors in modal typically remain brilliant and strong. Graying, as with $100 \%$ 
cotton textiles, does not occur ${ }^{3,4}$. They are also wear resistant and strong while maintaining a soft, silky feel. Modal fibers have found a wide variety of uses in clothing, outerwear and household furnishings ${ }^{5,6}$. They are often blended with cotton, wool or synthetic fibers and allow easy tone-in-tone dyeing ${ }^{7,8}$. In this study, modal fabrics (woven and knitted) are selected and conventionally pretreated to get rid of the basic impurities. The pre-treated fabrics were then subjected with $98 \%$ formic acid in different concentrations. The formic acid treated modal fabrics were then undergone for different testings, such as, physical properties, wicking, K/S values, fastness properties, SEM and FTIR analysis. These treatments on modal fabrics were correspondingly compared with those of cotton for its effectiveness.

\section{MATERIALS AND METHODS}

The textile fabrics used in this study were in the following descriptions:

I) Woven Fabrics: a) Modal (100\%) : yarn count (both warp and weft) - 27, GSM - 146, ends per inch - 84, picks per inch - 94; b) Cotton (100\%) : yarn count (both warp and weft) - 30, GSM - 137.2, ends per inch -94, picks per inch - 74; and II) Knitted Fabrics: a) Modal (100\%) : yarn count - 27.5, GSM 137, loop length-2.6 mm, b) Cotton (100\%) : yarn count - 30, GSM - 142, loop length-2.6 mm.

Natural dyes [annatto (bixa orellana), onion (allium cepa), pomegranate (punica granatum), indigo (indigofera tinctoria), myrobalan (terminalia chebula), bar berry (berberis vulgaris)] and synthetic dyes [reactive dye (reactive red HB - C.I. No. Red 24), and sulphur dye (sulphur black - C.I. No. sulphur Black 1)] used were in the commercial grade. The chemicals mentioned elsewhere for this study were in AR grade.

\section{Treatment on modal and cotton fabrics}

The modal and cotton fabrics (woven and knitted) were pretreated (desizing (for woven), scouring and bleaching) as per the established technique ${ }^{9,10,11}$. The pre-treated modal and cotton fabrics were subjected with $98 \%$ formic acid with the concentration of $1 \%, 2 \%, 3 \%, 4 \% \& 5 \%$ (owm) for 30 minutes at ambient temperature $\left(27^{\circ} \mathrm{C}\right)$.

\section{Measurement of physical properties of modal and cotton fabrics}

The physical properties such as tensile strength $^{9}$, stiffness ${ }^{12}$, crease recovery angle ${ }^{13,14}$ and mean drape coefficient of woven fabric ${ }^{15}$; and bursting strength and mean drape coefficient of knitted fabric ${ }^{15,16}$ of the modal and cotton fabrics was measured by the standard established methods.

\section{Wicking of modal and cotton fabrics}

Absorbency is the time taken for a water drop to penetrate into the textile material. The wettability of modal and cotton fabrics was determined as per AATCC test method $79^{17}$. The wicking height of the modal and cotton fabrics was determined ${ }^{18,19}$. Fabric samples measuring $10 \mathrm{~cm}$ y! $2.5 \mathrm{~cm}$ were taken. Each of the sample pieces was clamped to a scale and held at a position such that the tip of the sample just touched the water taken in a beaker. $1 \%$ reactive dye (Reactive Red M8B, Cl No.: Reactive Red 11) was added for tracking the movement of water. The height of water reached after five minutes was measured ${ }^{20}$.

\section{Dyeing and K/S analysis of modal and cotton fabrics}

The dyeability of modal and cotton fabrics (woven and knitted) was investigated using natural and synthetic dyes. Dyeing was carried out at boil for two hours with a material to liquor ratio of 1:20 as per the established technique of dyeing for natural and synthetic dyes. The dyed samples were washed, soaped and dried ${ }^{10,21,22}$. Colorimetric data of natural and synthetic dyed modal and cotton fabrics were determined by AATCC Test Method $-135^{23}$ using a Datacolor SF 600 plus spectrophotometer interfaced to a PC. Measurements were taken regarding colour presence, brightness, dullness and colour intensity with the specular component of the light excluded and the UV component included using illuminant D65 and $10^{\circ}$ standard observer. Each fabric was folded once so as to give two thickness and average of five readings were taken each time.

\section{Color fastness analysis of the modal and cotton fabrics}

The natural and synthetic dyed samples were washed under condition IIIA of AATCC Test Method 124-2001 to determine the color change 


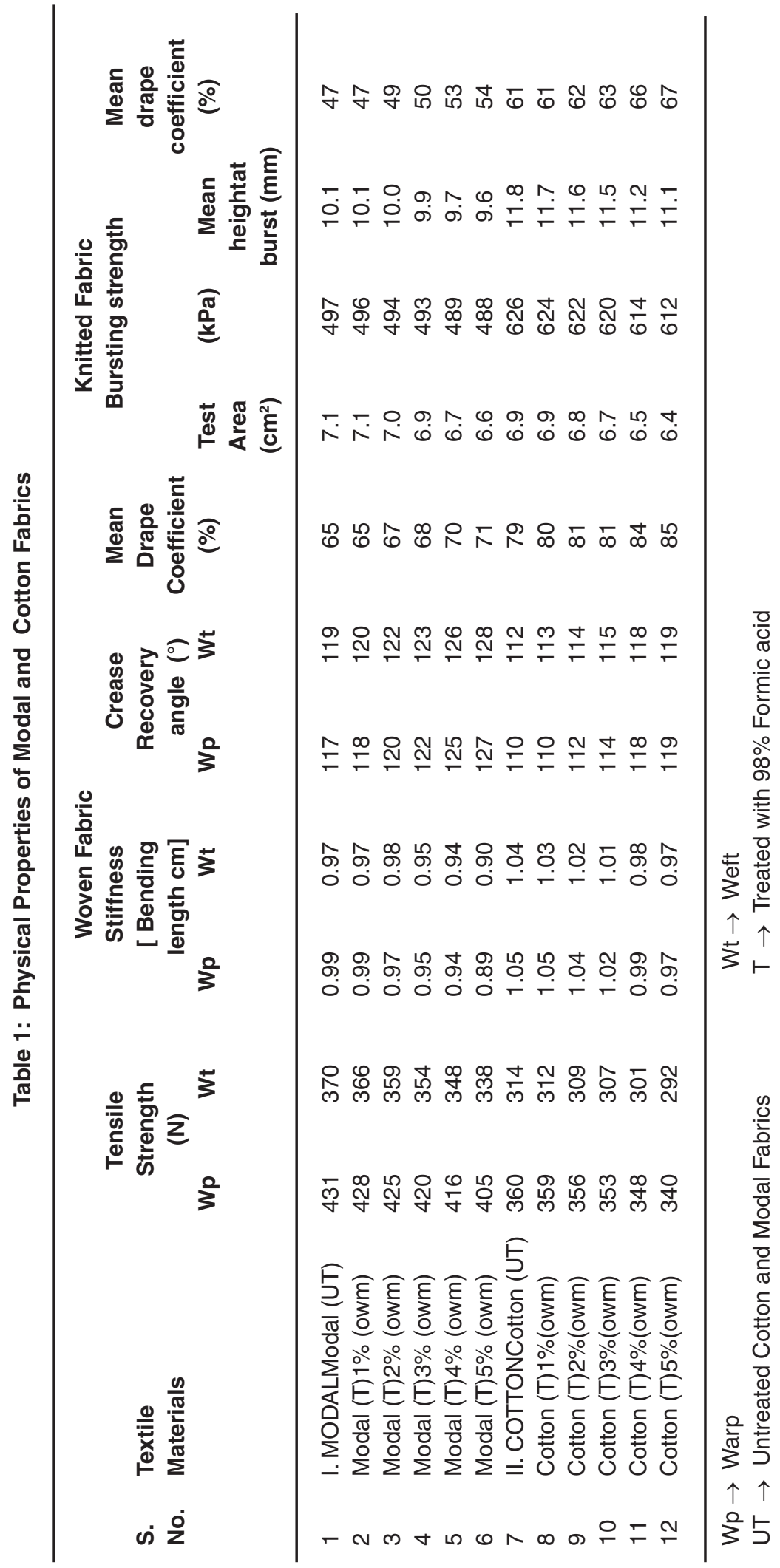


effect of dyed fabrics ${ }^{24,25}$. Light fastness tests (AATCC, 2003), were carried out according to AATCC Test Method $16 \mathrm{E}-1998^{26}$. The samples were exposed to 5, 10 AFUs (AATCC Fading Unit) to determine the color change AATCC $16-1998^{27}$. AATCC standardized crock meter was used to determine the rubbing fastness of natural dyed fabrics under wet and dry condition to assess the color change and staining property AATCC 61-1996 ${ }^{28}$.

Table 2: Wicking of Modal and Cotton Fabrics

\begin{tabular}{llcc}
\hline S. & Textile Materials & \multicolumn{2}{c}{ Wicking (cm) } \\
No. & & Woven & Knitted \\
\hline 1 & I. MODALModal (UT) & 13 & 11 \\
2 & Modal (T)1\% (owm) & 14 & 12 \\
3 & Modal (T)2\% (owm) & 15 & 12 \\
4 & Modal (T)3\% (owm) & 16 & 13 \\
5 & Modal (T)4\% (owm) & 19 & 16 \\
6 & Modal (T)5\% (owm) & 20 & 17 \\
7 & Il. COTTONCotton (UT) & 14 & 13 \\
8 & Cotton (T)1\%(owm) & 15 & 14 \\
9 & Cotton (T)2\%(owm) & 16 & 15 \\
10 & Cotton (T)3\%(owm) & 17 & 15 \\
11 & Cotton (T)4\%(owm) & 19 & 18 \\
12 & Cotton (T)5\%(owm) & 20 & 19 \\
\hline
\end{tabular}

\section{SEM study of modal and cotton fabrics}

The surface morphology of modal and cotton fabrics was observed in SEM (JOEL JSM6360 model microscope, Japan $)^{29,30}$

\section{FTIR study of modal fabrics}

Fourier Transfer Infra Red (FTIR) spectrophotometer (Shimadzu, Japan) was used to analyze the functional groups of the dyed modal fabrics $^{31,32}$.

\section{RESULTS AND DISCUSSION}

\section{Physical properties of modal and cotton fabrics}

The physical properties such as tensile strength, stiffness, crease recovery angle and mean drape coefficient of woven fabric; and bursting strength and mean drape coefficient of knitted fabric for modal and cotton fabrics are shown in Table 1. The modal fabric shows higher values of tensile strength (431 N (warp) and $370 \mathrm{~N}$ (weft) - untreated) and crease recovery angle (117 (warp) and 119 (weft) - untreated) respectively both in warp and in weft directions compared to that of the cotton fabric. However, the stiffness $(0.99 \mathrm{~cm}$ (warp) and 0.97 $\mathrm{cm}$ (weft) - untreated) and mean drape co-efficient $(65 \%)$ are less in modal fabric over that of cotton fabric. In the case of knitted fabric, cotton shows greater values than that shown by modal fabric

Table 3: K/S values of Modal and Cotton Fabrics

\begin{tabular}{|c|c|c|c|c|c|c|}
\hline \multirow[t]{3}{*}{ S.No. } & \multirow[t]{3}{*}{ Dyes } & \multirow{3}{*}{$\begin{array}{l}\text { Colours } \\
\text { obtained }\end{array}$} & \multicolumn{4}{|c|}{$\mathrm{K} / \mathrm{S}$ values of the dyed fabrics } \\
\hline & & & \multicolumn{2}{|c|}{ Modal } & \multicolumn{2}{|c|}{ Cotton } \\
\hline & & & Woven & Knitted & Woven & Knitted \\
\hline 1 & Annatto & Orange & 13.98 & 13.10 & 13.72 & 12.77 \\
\hline 2 & Onion & Orange Red & 14.50 & 13.17 & 13.70 & 12.98 \\
\hline 3 & Pomogranate & Brown & 14.47 & 13.52 & 13.76 & 12.84 \\
\hline 4 & Indigo & Blue & 14.60 & 14.33 & 14.01 & 13.45 \\
\hline 5 & Myrobalan & Green & 14.45 & 13.62 & 13.66 & 13.76 \\
\hline 6 & Bar berry & Yellow & 14.54 & 13.41 & 13.95 & 13.32 \\
\hline 7 & Reactive Dye & Red & 15.07 & 14.95 & 14.42 & 14.25 \\
\hline 8 & Sulphur Dye & Black & 14.65 & 14.32 & 14.21 & 13.65 \\
\hline \multicolumn{3}{|c|}{$\begin{array}{l}\text { No Formic acid treatment } \\
\text { (Reactive dye) : Red }\end{array}$} & 12.12 & 12.84 & 12.95 & 12.62 \\
\hline
\end{tabular}




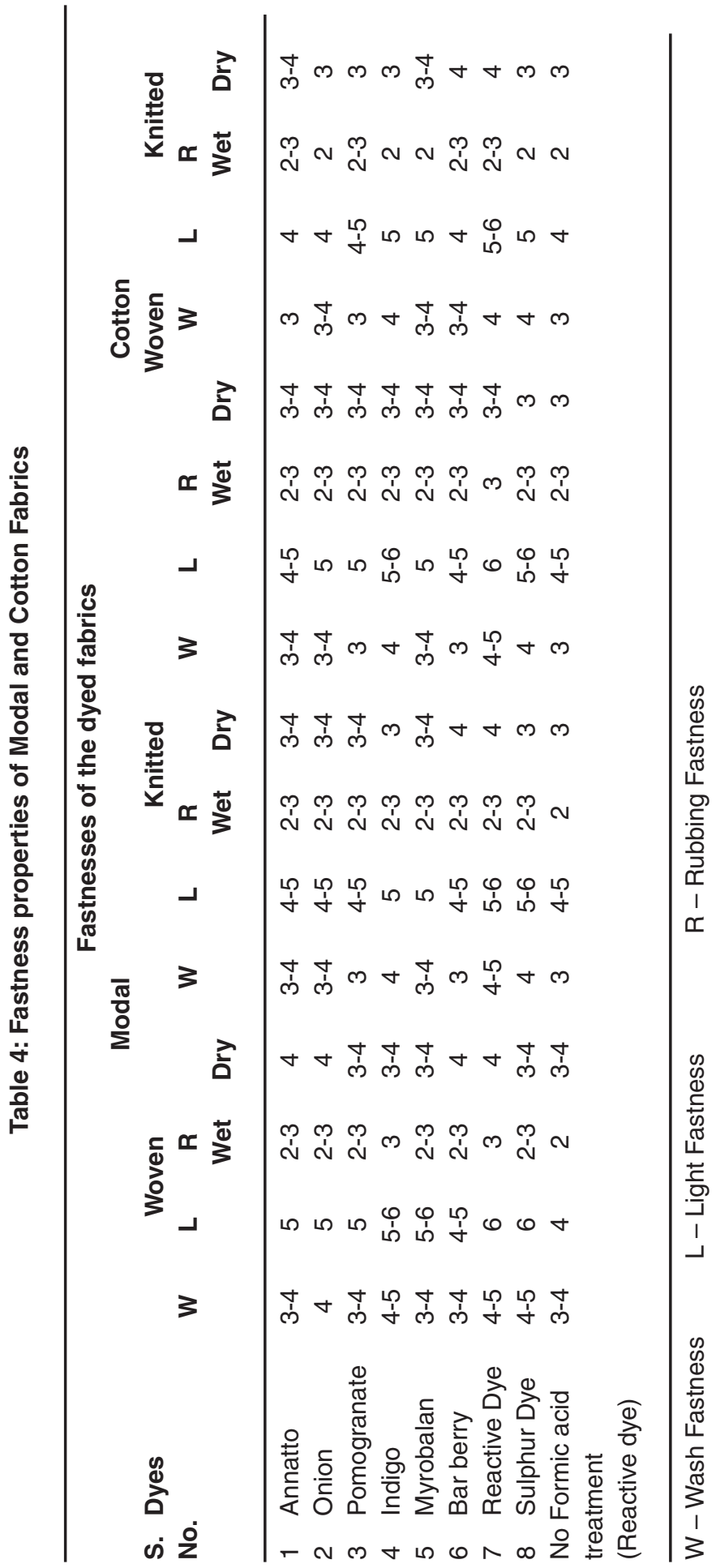


for bursting strength and mean drape coefficient respectively. The modal fabric after treatment with formic acid $(98 \%)$ at different concentrations for 30 minutes at room temperature show changes in the physical properties. The tensile strength and stiffness of formic acid treated modal fabric are reduced marginally upto the formic acid treatment of $4 \%$ (owm), and at 5\% (owm) the loss is significant similar to that of cotton fabric. However, the crease recovery and mean drape co-efficient of formic acid treated modal fabric is increased considerably up to the concentration of $4 \%$ (owm), and at $5 \%$ (owm) it is only marginal. In the case of knitted modal fabric the bursting strength is decreased correspondingly with respect to the concentration of formic acid, while the mean drape co-efficient is increased considerably upto $4 \%$ (owm).

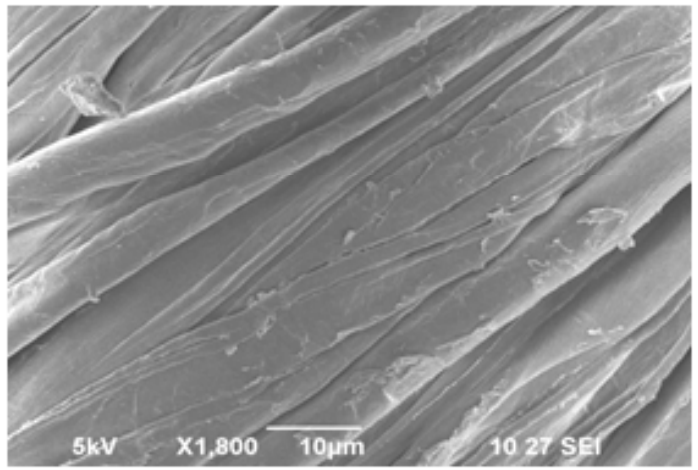

1-a) No formic acid treated \& Indigo dyed woven modal fabrics

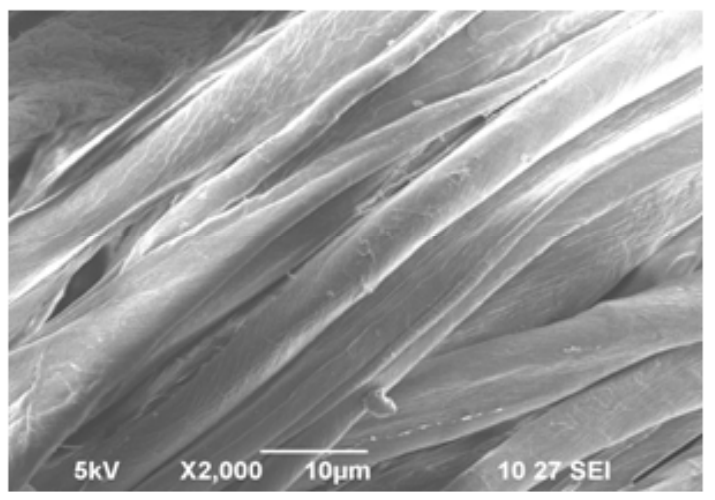

1-c) No formic acid treated \& Indigo dyed Knitted modal fabrics

\section{Wicking of modal and cotton fabrics}

The data of wicking behaviour of modal and cotton (woven and knitted) fabrics are given in Table 2. From table 2, it is clear that the wicking behaviour of all the samples is good, however woven fabrics show some increase over the knitted fabrics. The wicking behaviour shown by cotton is higher than that of modal fabrics both in the case of woven and knitted fabrics. The modal fabric after treatment with formic acid (98\%) at different concentrations for 30 minutes at room temperature shows improved wicking compared with the untreated one. The increase in wicking is significant upto $4 \%$ (owm) formic acid treatment, and afterwards the increase is only marginal. The similar trend is also observed for cotton fabrics.

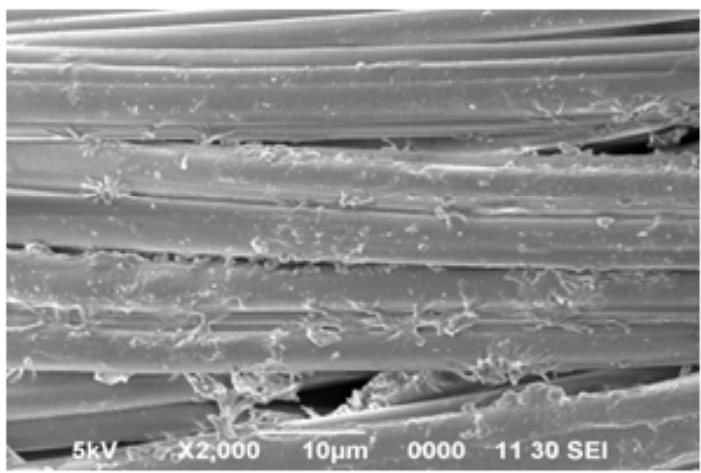

1-b) Formic acid treated \& Indigo dyed

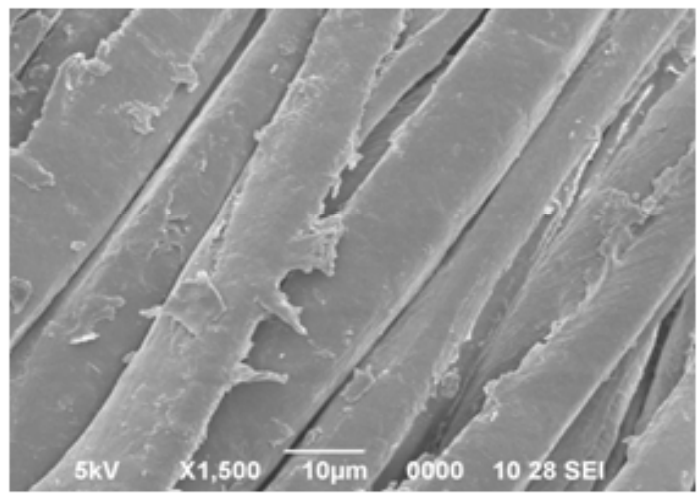

1-d) Formic acid treated \& Indigo dyed

Fig. 1: Formic acid treated and indigo dyed modal fabrics 


\section{K/S values of Modal and Cotton Fabrics}

The K/S values of the dyes such as annatto, onion, pomogranate, indigo, myrobalan, barberry, reactive dye, and sulphur dye applied on formic acid treated modal and cotton (woven and knitted) fabrics are given in Table 3. The formic acid treatment was identified effective at the concentration of $4 \%$ (owm), hence that condition was considered as optimum parameter. For dyeing, the modal and cotton fabrics were given formic acid treatment only with $4 \%$ (owm) concentration for 30 minutes at ambient temperature. From this table it is observed that woven fabrics show overall high $\mathrm{K} / \mathrm{S}$ values than the knitted fabrics. The K/S value of formic acid treated modal fabric is high when compared those of the cotton fabrics (woven and knitted). The higher $\mathrm{K} / \mathrm{S}$ value on the formic acid treated modal fabric is influenced by the higher swelling action of formic acid treatment.

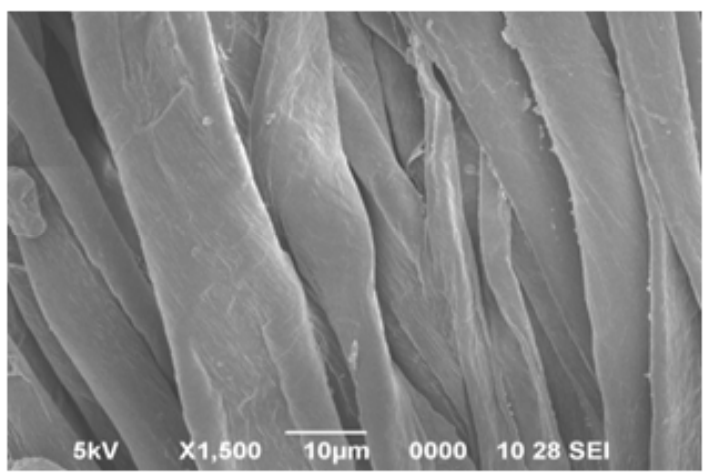

2-a) No formic acid treated \& Indigo dyed woven cotton fabrics

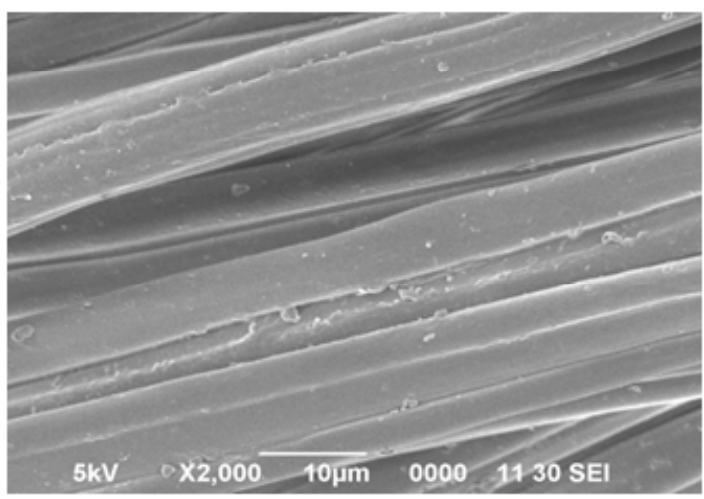

2-c) No formic acid treated \& Indigo dyed Knitted cotton fabrics
Among the dyes applied on the formic acid treated modal fabrics, there is only a marginal differences in the K/S values; however reactive dye shows the maximum $\mathrm{K} / \mathrm{S}$ values. Even though the woven and knitted modal and cotton fabrics posses only a small differences in the K/S values for the dyes (annatto, onion, pomogranate, indigo, myrobalan, bar berry, reactive dye, and sulphur dye) applied on these fabrics; there is a uniform trend maintained in these values and the values of woven fabric give an edge over the knitted fabric. The untreated (no formic acid) modal and cotton fabrics were dyed only with the representative dye (reactive). From the table 3 , it is evident that the overall K/S values of formic acid ( $4 \%$ owm) treated modal and cotton fabrics are significant compared with that of the untreated dyed fabrics.

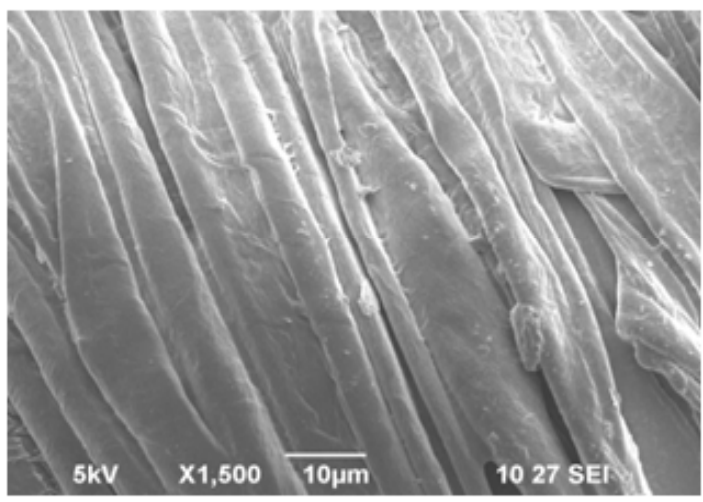

2-b) Formic acid treated \& Indigo dyed

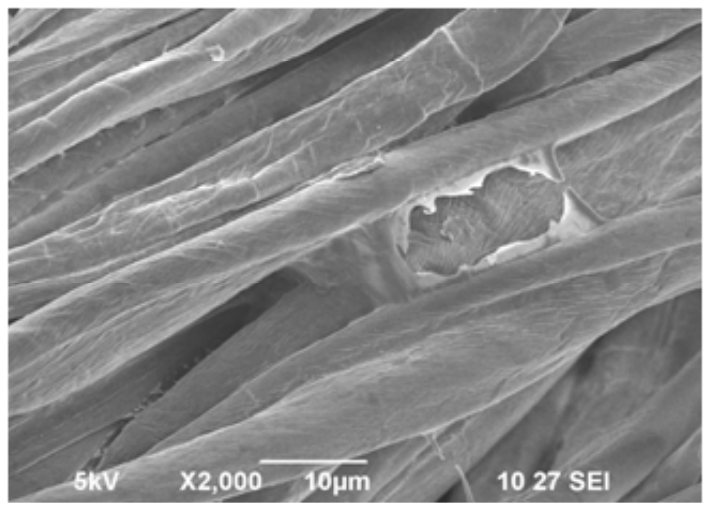

2-d) Formic acid treated \& Indigo dyed

Fig. 2: Formic acid treated and indigo dyed cotton fabrics 
1

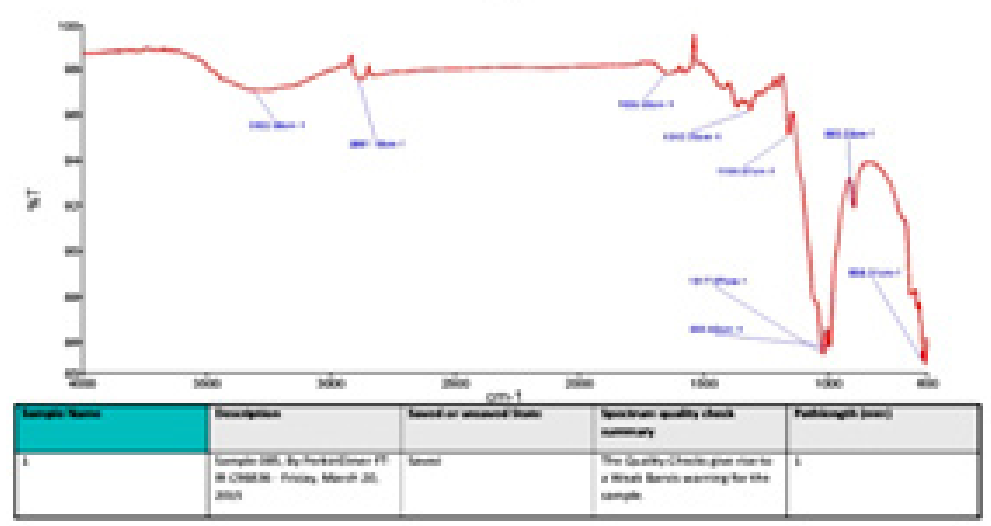

3-a) No formic acid treated \& Indigo dyed

2

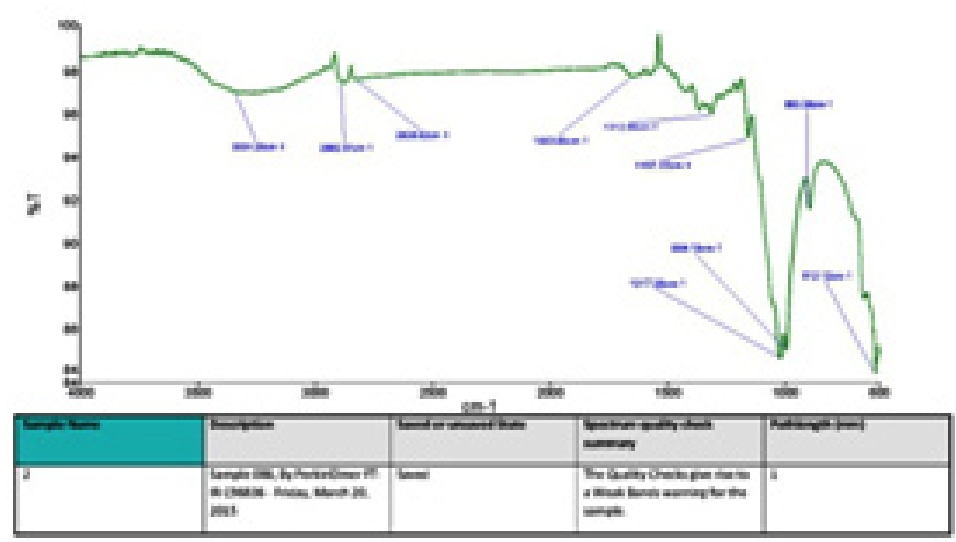

3-b) Formic acid treated (1\%) \& Indigo dyed modal fabrics

12

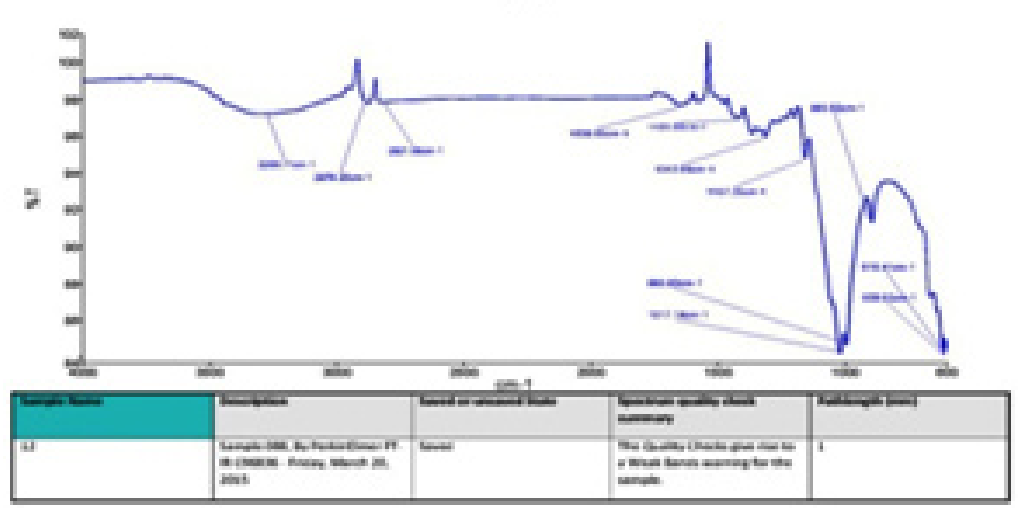

3-c) Formic acid treated (4\%) \& Indigo dyed 


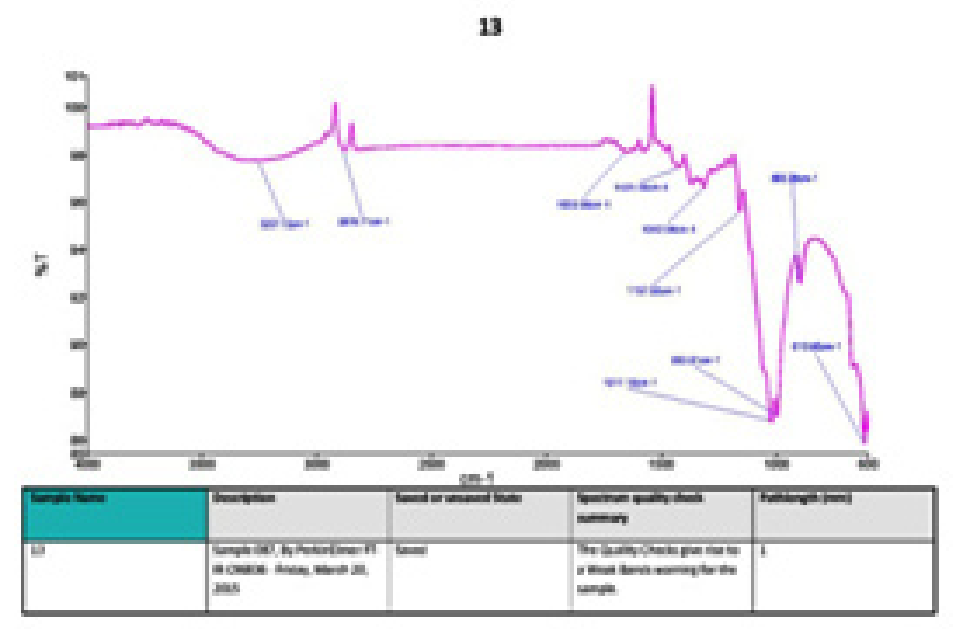

3-d) Formic acid treated (5\%) \& Indigo dyed modal fabrics

Fig. 3: Formic acid treated and indigo dyed modal fabrics

\section{Fastness properties of Modal and Cotton Fabrics}

The fastness properties (wash, light and rubbing) of natural and synthetic (indigo, kum kum, bar berry, reactive dye $(\mathrm{M})$, reactive dye $(\mathrm{H})$ and sulphur) dyes applied on formic acid treated (4\% owm) modal and cotton fabrics (woven and knitted) are given in Table 4. The wash fastness of the formic acid treated modal fabrics is good as compared with other fatnesses like light and rubbing fastness properties. The good wash fastness property is due to the strong polymeric reaction of the fabric materials and the also of the dye. It is obvious that the light fastness and rubbing fastness properties are moderate to poor only due to their behaviour towards these applications. From this table 4, it is also evident that the overall fastness properties of the formic acid treated and dyed modal fabrics are higher than that of the no formic acid treatment fabrics. The similar trend is also seen for cotton fabrics.

\section{SEM Analysis of Modal and Cotton Fabrics}

The analysis of SEM images of formic acid treated and dyed modal and cotton fabrics are given in the representative Figures $1 \& 2$. Figure 1 reveals about the micrographs of formic acid treated and dyed modal fabrics. Figure 2 reveals about the micrographs of formic acid treated and dyed cotton fabrics. These figures show clearly about the effect of formic acid in the corresponding materials and the subsequent dyeing. There is a clear difference between the no formic treated \& dyed; and formic acid treated \& dyed (indigo) modal fabrics (woven and knitted). Similar trend is also seen for the cotton fabrics. These micrographs reveal about the enhancement of dyeing after formic acid treatment on modal and cotton fabrics respectively.

\section{FTIR study of indigo dyed modal fabrics}

The modal fabrics are dyed with different dyes. However as representation only indigo dyed woven modal fabric is selected for FTIR study. The indigo dyed modal fabrics are studied and their FTIR results are shown in Figure 3. In Figure 3a, the FTIR graph of the modal fabric without any formic acid treatment is seen. Subsequently the FTIR graphs of the indigo dye after application on the formic acid treated $(1 \%, 4 \%, \& 5 \%)$ woven modal fabrics are given in the Figures $3 b, 3 c$, and $3 d$ respectively. The FTIR graphs for this indigo dyed modal fabrics are analyzed as per the following data in Table 5. The FTIR graph of $1 \%$ formic acid treated modal fabric when compared with that of no formic acid treatment modal fabric there is no difference between them. However, Figure 3c, the 4\% formic acid treated modal fabric gives a considerable change in the FTIR graph [33] when compared with Figures $3 a$ and $3 b$. When the concentration of formic acid is increased beyond $4 \%$ there is no change in the FTIR graph as seen in Figure $3 d$. 
Table 5: Standard data for the analysis of FTIR graph

\begin{tabular}{lll}
\hline S.No. & Peak range $\left(\mathbf{c m}^{-1}\right)$ & Functional groups \\
\hline 1 & $3600-3700$ & Non bonded hydroxyl group-OH- \\
2 & $3300-3400$ & Alkyne -C-H- stretch \\
3 & $3200-3300$ & Hydroxyl group ( H-bonded -OH- stretch ) \\
4 & $3000-3200$ & Aromatic ring $(-\mathrm{C}-\mathrm{H}$ - stretch) \\
5 & $2900-3000$ & Methylene $-\mathrm{CH}$ - stretch \\
6 & $2700-2800$ & Terminal aldehyde $-\mathrm{CH}$ - stretch \\
7 & $2600-2700$ & Hydrogen bonded -OH- group \\
8 & $2500-2600$ & Thiols (-S-H- stretch) \\
9 & $2400-2500$ & -CH- stretch of aromatic compounds \\
10 & $2300-2400$ & -OH-stretching of Carboxylic acid \\
11 & $2200-2300$ & Cyano compounds,disubstituted alkynes \\
12 & $2100-2200$ & C-triple bond-C- stretch \\
13 & $2000-2100$ & Cyanide ion and related ion \\
14 & $1800-2000$ & Transition metal carbonyl group \\
15 & $1700-1800$ & Carbonyl group \\
16 & $1600-1700$ & -C-double bond-C stretch \\
17 & $1500-1600$ & Aromatic ring stretch-NH-bend \\
18 & $1400-1500$ & Organic Sulphates \\
19 & $1300-1400$ & -OH- bend \\
20 & $1200-1300$ & Aromatic primary amine -CN- stretch \\
21 & $1100-1200$ & Secondary amine -CN- stretch \\
22 & $1000-1100$ & -C-C- stretch \\
23 & $900-1000$ & Cyclo hexane ring vibrations \\
24 & $800-900$ & Peroxides -C-O-O-stretch \\
25 & $700-800$ & Skeletal -C-C- vibrations \\
26 & $600-700$ & Aliphatic Bromo compounds \\
27 & $500-600$ & -C-I- stretch \\
\hline
\end{tabular}

\section{CONCLUSIONS}

The tensile strength of modal fabric both in warp and weft direction and crease recovery angle are very good in comparison with cotton fabric. Whereas the stiffness [warp \& weft] and mean drape co-efficient are less in modal fabric over that of cotton fabric. In the case of knitted fabric, cotton shows greater values than that shown by modal fabric for bursting strength and mean drape coefficient respectively. The modal fabric after treatment with formic acid (98\%) at the concentration of $4 \%$ (owm) for 30 minutes at room temperature gives considerable changes in the physical properties under the favorable limit suitable for the application. The wicking is improved in the modal fabric (woven and knitted) after treatment with formic acid (98\%) at the concentration of $4 \%$ (owm) for 30 minutes at room temperature similar to that of cotton fabric. The K/S values of the dyes such as annatto, onion, pomogranate, indigo, myrobalan, barberry, reactive dye, and sulphur dye applied on formic acid treated modal (woven and knitted) fabrics are good compared with that of no formic acid treated one, similar to the trend of cotton fabrics. The fastness properties (wash, light, \& rubbing) of the formic acid treated and dyed (annatto, onion, pomogranate, indigo, myrobalan, bar berry, reactive dye and sulphur dye) woven and knitted modal fabrics are comparatively good over that of no formic acid treated one, as that of cotton fabrics. In general, the overall fastness properties are more in formic acid treated fabrics. SEM micrographs reveal that the formic acid treated \& dyed modal and cotton fabrics 
give good dyeing effect compared with that of no formic acid treated one. FTIR graphs reveal that the formic acid treatment of $1 \%$ gives no effect whereas $4 \%$ formic acid gives considerable changes on the modal fabric; after $4 \%$ of formic acid treatment the effect is negligible only.

\section{ACKNOWLEDGEMENTS}

The authors wish to thank the Management and Principal, PSG College of Technology, Coimbatore for given the permission and providing the necessary infrastructure. The authors also thank The Head, Department of Applied Science, PSG CT for the kind help rendered in the completion of this work.

\section{REFERENCES}

1. Heinze, T.; Liebert, T. Unconventional methods in cellulose Functionalization. Progress in Polymer Science. 2001, 26(9), 1689-1762.

2. Saalwachter, K.' Burchard, W. Cellulose in new metal-complexing solvents: Semidilute behavior in Cd-tren; Macromolecules.2001, 34(16), 5587-5598.

3. Lewin, M. Handbook of Fibre Chemistry; 3rd edition, (CRC Press, Baco Raton, USA), 2007, 331-382.

4. Teli, M.D.; Kumar, G.V.N.S. Functional textiles and apparels - Technical Textile, Journal of the Textile Association. 2007, 21-30.

5. Schurz, J. Trends in polymer science. A bright future for cellulose, Progress in Polymer Science. 1999, 24(4), 481-483.

6. Bredereck, K.; Hermanutz, F. Man-made cellulosics. Review of Progress in Coloration and Related Topics. 2005, 35, 59-75.

7. Simpson, V. India's Textile and apparel industry: Growth potential and trade and investment opportunities (Staff Research Study, Office of Industries, U. S. International Trade Commission). Washington, USA. 2011.

8. Lewin, M.; Sello. Handbook of Fiber Science and Technology, Vol. II, Part B, Dekker Series, New York, USA. 2000, 120-125.

9. Saville, B.P. Physical Testing of Textiles; Wood Head Publishing Limited and CRC Press, Cambridge, England. 2004, 205-210.

10. Trotman, E.R. Dyeing and Chemical Technology of Textile Fibers: Sixth edition, Edward Arnold, London. 1984, 187-217.

11. Shukla, S.R. Advances in preparatory process in cotton; NCUTE-Programme Series: Chemical Preparatory Process in Textiles,
Indian Institute of Technology -Delhi; New Delhi, 2000, 85-92.

12. ASTM Test Method 1388-96: Standard Test Method for Stiffness of Fabrics: Annual Book of ASTM Standards, Vol.07.01, West Conshohocken, PA, USA, 2001.

13. AATCC Test Method 66-2003: Wrinkle recovery of woven fabrics recovery Angle: Technical Manual of the AATCC, Research Triangle Park, USA, 2003.

14. Connell, D.L. The Control of Shrinkage in Textile Finishing (Editor Heywood D): Woodhead Publication Ltd., Cambridge, England. 2005, 60-62.

15. BSI, BS 5058:1973, British Standard Method for the Assessment of Drape of Fabrics, BS Handbook : London. 1990.

16. Collier, B.J.; Epps, H.H.; Textile Testing and analysis: $6^{\text {th }}$ edition, Prentice Hall Inc, New Jersey, USA. 1999, 30-35.

17. AATCC Technical Manual, American Association of Textile Chemists and Colorists, Research Triangle Park, N.C. 1991, 66.

18. Kissa, E. Wetting and wicking; Textile Research Journal. 1996, 66(10), 660-668.

19. Tyagi, G.K.; Krishna, G.; Bhattacharya, S.; Kumar, P. Comfort aspects of finished polyester-cotton and polyester-viscose ring and MJS yarn fabrics; Indian Journal of Fiber \& Textile Research. 2009, 34, 137-143.

20. Swarna Natarajan.; Jeyakodi Moses, J. Surface Modification of Polyester Fabric using Polyvinyl in Alkaline Medium;, Indian Journal of Fiber \& Textile Research. 2012, 37, 287- 291.

21. Mohanty, B.C.; Chandramauli, K.V.; Naik, H.D. Natural Dyeing Process of India: Published by Calico Museum of Textiles, India, 1987. 
22. Gulrajani, M.L.; Gupta, D. Natural Dyes and their Application to Textiles: Indian Institute of Technology -Delhi; New Delhi, 1992.

23. AATCC Test Method 135-1985; Colour measurement of textiles: Instrumental Technical Manual of the AATCC, Research Triangle Path, USA, 2003.

24. AATCC test method 61, 2(A)- 2001; Colour Fastness to Laundering, Home and Commercial: Technical Manual of the AATCC, Research Triangle Park, U.S.A., 2001.

25. BIS Test Method IS:764-1979; Test 3, Indian Standard Method for Determination of Colour Fastness of Textile Materials to Washing, Bureau of Indian Standards, 1979.

26. AATCC Test Method 16-1998; Colour Fastness to Light: Technical Manual of the AATCC, Research Triangle Park, USA, 2003.

27. AATCC test method 8-2007; Colour Fastness to Crocking: Technical Manual of the AATCC,
Research Triangle Park, USA, 2007.

28. AATCC Test Method 61-1996; Colour Fastness to Laundering: Home and Communication Accelerated: Technical manual of the AATCC, Research. Triangle Park, U.S.A, 2003.

29. Hearle, J.W.S. Use of the Scanning Electron Microscope (Pergamon Press, Oxford), 1972.

30. Wenbin L.; Zengbo L. Biological Stability and Antimicrobial Activity Analysis of Antagonism Actinomycete SC-04 Fermentation Fluid. Orient J Chem, 2016, 32(1).

31. John Coates, Encyclopedia of Analytical Chemistry, 2000, 12, 10815 - 10837.

32. Sawyer, D.T.; Heineman, W.R.; Beebe, J.M. Chemistry Experiments for Instrumental Methods, John Wiley \& Sons, USA, 1984.

33. Srivastava, S.; Gadhave, R. Furfural Hydrogenation on Alloyed Copper Catalysts With Additives of Ferrosilicium. Orient J Chem 2015, ;31(4). 